\title{
Chaos expansions and Malliavin calculus for Lévy processes
}

Josep Lluís Solé, Frederic Utzet, and Josep Vives

Departament de Matemàtiques, Facultat de Ciències, Universitat Autónoma de
Barcelona, 08193 Bellaterra (Barcelona), Spain. jllsole@mat.uab.cat,
utzet@mat.uab.cat and vives@mat.uab.cat. *

Summary. There are two different chaos expansions of a square integrable functional of a Lévy process: one proved by Itô [9] and the other by Nualart and Schoutens [17]. Related to each expansion a Malliavin type Calculus has been developed, being both quite different. In this paper we review the relationship between both approaches, and compare the corresponding Clark-Ocone-Haussmann representation formula.

\section{Introduction}

Kyoshi Itô, in his paper of 1956 [9], proved a chaotic representation of any square integrable functional of a Lévy process. However, in the Brownian motion case (Itô [8]) (and also in the Poisson process case, see, for example, [18]), the representation is expressed in terms of multiple integrals with respect to Brownian motion (respectively, the Poisson process), whereas in the general Lévy case it is necessary to introduce a two-parameter random measure associated with the Lévy process and the representation of a functional is written using multiple integrals with respect this random measure. On the other hand, Nualart and Schoutens [17], under some exponential integrability conditions on the Lévy measure, give a kind of chaotic representation expressed, roughly speaking, in terms of iterated integrals with respect the powers of the jumps of the Lévy processes. Both approaches enable the construction of a Malliavin calculus type for Lévy processes.

To be more specific, the Itô representation gives a Fock space structure to $L^{2}(\Omega)$, and then it is natural to define a two parameter (time and space) derivative operator $D_{t, x}$ as an annihilator operator, and a Skorohod integral $\delta$ of a two parameter process as a creation operator. These ideas have been developed by several authors: see Benth et al. [2], Øksendal and Proske [21], Di Nunno et al. [6, 7], Løkka [13] (all these authors using a different random

\footnotetext{
* Supported by grants BFM2003-00261 Ministerio de Educación y Ciencia and 2001SGR-00174 Generalitat de Catalunya.
} 
measure than Itô [9]); see also Lee and Shih $[10,11]$ for a pure white noise approach (with the Itô random measure), Privault [23], in the context of quantum probability, and Sole et al. [26].

In the Nualart and Schoutens approach, a family of strongly orthogonal martingales is introduced and the representation is expressed in terms of iterated integrals with respect these martingales; then, we can define a (one parameter) derivative operator for each $n, D_{t}^{(n)}$, as an annihilation operator of the $n$-th martingale, and a Skorohod integral $\delta^{(n)}$ as the adjoint of Dom $D^{(n)}$ that turns out to be a creation operator for the martingale $n$ : see Leon et al. [12] and Davis and Johansson [3].

In this paper we review some of the above cited papers, we extend some results proved only for purely jumps process or for simple Lévy processes to a general Lévy process with both continuous and jump part, and we study in deep the relationships between both approaches; in particular, we study the Clark-Ocone-Haussmann representation formula in both contexts.

\section{Itô chaotic calculus for Lévy processes}

We will restrict ourselves to a square integrable Lévy processes because of the main results that we study need this hypothesis; for the same reason, we will only consider processes on a finite time interval $[0, T]$. This simplifies the definitions and notations; however, we should point out that Itô [9] proved the chaotic representation property for a completely general Lévy process with index on $\mathbb{R}$, and also the definition of the Malliavin derivative and the Skorohod integral can be done without the square integrability hypothesis (see Sole et al. [26]).

\subsection{Itô multiple integrals}

Consider a complete probability space $(\Omega, \mathcal{F}, \mathbb{P})$ and let $X=\left\{X_{t}, t \in[0, T]\right\}$

be a Lévy process (that means, $X$ has stationary and independent increments, is continuous in probability and $X_{0}=0$ ), cadlag, centered, with $\mathbb{E}\left[X_{1}^{2}\right]<\infty$, and with Lévy measure $\nu$. For all these concepts we refer to Sato [24]. Note that the square integrability of $X_{1}$ implies that $\int_{\mathbb{R}} x^{2} \nu(d x)<\infty$. Write $\mathbb{R}_{0}=$ $\mathbb{R}-\{0\}$. The process $X$ can be represented as

$$
X_{t}=\sigma W_{t}+\iint_{(0, t] \times \mathbb{R}_{0}} x d \widetilde{N}(t, x),
$$

where $\left\{W_{t}, t \geq 0\right\}$ is a standard Brownian motion and $\sigma \geq 0$,

$$
N(B)=\#\left\{t:\left(t, \Delta X_{t}\right) \in B\right\}, \quad B \in \mathcal{B}\left([0, T] \times \mathbb{R}_{0}\right),
$$

is the jump measure of the process, where $\Delta X_{t}=X_{t}-X_{t-}$, and 


$$
d \widetilde{N}(t, x)=d N(t, x)-d t d \nu(x)
$$

is the compensated jump measure.

Itô [9] extends the process $X$ to an independent random measure $M$ on $([0, T] \times \mathbb{R}, \mathcal{B}([0, T] \times \mathbb{R}))$ : for a set $E \in \mathcal{B}([0, T] \times \mathbb{R})$, define

$$
M(E)=\sigma \int_{E(0)} d W_{t}+\iint_{E^{\prime}} x d \widetilde{N}(t, x)
$$

where $E(0)=\left\{t \in \mathbb{R}_{+}:(t, 0) \in E\right\}$ and $E^{\prime}=E-\{(t, 0) \in E\}$. Then, for $E_{1}, E_{2} \in \mathcal{B}([0, T] \times \mathbb{R})$

$$
\mathbb{E}\left[M\left(E_{1}\right) M\left(E_{2}\right)\right]=\mu\left(E_{1} \cap E_{2}\right),
$$

where $\mu$ is the measure on $\mathcal{B}([0, T] \times \mathbb{R})$ given by

$$
\mu(E)=\sigma^{2} \int_{E(0)} d t+\iint_{E} x^{2} d t d \nu(x) .
$$

As in the Brownian setup, it is essential that this measure is continuous (Itô [9], pag. 256).

The following step is to construct multiple integrals with respect to $M$, and that is done in the same way as in the Brownian case: First consider an elementary function of the form

$$
f=\mathbf{1}_{E_{1} \times \cdots \times E_{n}},
$$

where $E_{1}, \ldots, E_{n} \in \mathcal{B}\left(\mathbb{R}_{+} \times \mathbb{R}\right)$, are pairwise disjoints, with

$$
\mu\left(E_{1}\right)<\infty, \ldots, \mu\left(E_{n}\right)<\infty,
$$

and let

$$
I_{n}(f)=M\left(E_{1}\right) \cdots M\left(E_{n}\right) .
$$

By linearity and continuity, $I_{n}$ is extended to

$$
L_{n}^{2}=_{\operatorname{def}} L^{2}\left(([0, T] \times \mathbb{R})^{n}, \mathcal{B}([0, T] \times \mathbb{R})^{n}, \mu^{\otimes n}\right) .
$$

This integral has the usual properties (Itô [9], Theorem 1):

1.

where $\tilde{f}$ is the symmetrization of $f$ :

$$
I_{n}(f)=I_{n}(\widetilde{f}),
$$

$$
\widetilde{f}\left(z_{1}, \ldots, z_{n}\right)=\frac{1}{n !} \sum_{\pi \in \mathfrak{G}_{n}} f\left(z_{\pi(1)}, \ldots, z_{\pi(n)}\right),
$$

2 .

$z_{i}=\left(t_{i}, x_{i}\right), i=1, \ldots, n$ and $\mathfrak{G}_{n}$ is the set of permutations of $\{1,2, \ldots, n\}$. . $\quad I_{n}(a f+b g)=a I_{n}(f)+b I_{n}(g)$. 
3.

$$
\mathbb{E}\left[I_{n}(f) I_{m}(g)\right]=\delta_{n, m} n ! \int_{\left(\mathbb{R}_{+} \times \mathbb{R}\right)^{n}} \tilde{f} \widetilde{g} d \mu^{\otimes n},
$$

where $\delta_{n, m}=1$, if $n=m$, and 0 otherwise.

Let $\left\{\mathcal{F}_{t}^{X}, t \in[0, T]\right\}$ be the natural filtration of $X$ completed with the null sets of $\mathcal{F}$; it is well known that this filtration is right continuous. Write

$L^{2}(\Omega)=L^{2}\left(\Omega, \mathcal{F}_{T}^{X}, \mathbb{P}\right)$. Itô $[9$, Theorem 2], proves that every functional $F \in$ $L^{2}(\Omega)$ can be represented as

$$
F=\sum_{n=0}^{\infty} I_{n}\left(f_{n}\right), \quad f_{n} \in L_{n}^{2},
$$

and the representation is unique if we take every $f_{n}$ symmetric. We will always assume the symmetry in the kernels $f_{n}$ of such decompositions.

\subsection{Derivative operators}

From the chaotic representation property, it is natural to apply all the machinery of the annihilation operators (Malliavin derivatives) and creation operators (Skorohod integrals) on Fock spaces as is exposed in Nualart and Vives $[18,19]$. This point of view has been developed by Benth et al. [2], Øksendal and Proske [21], Di Nunno et al. [6, 7], Løkka [13], Yablonski [28], Lee and Shih [10,11] and Solé et al. [26].

Let Dom $D$ be the set of functionals $F \in L^{2}(\Omega)$ with chaotic representation $F=\sum_{n=0}^{\infty} I_{n}\left(f_{n}\right),\left(f_{n}\right.$ symmetric $)$ that satisfies

$$
\sum_{n=1}^{\infty} n n !\left\|f_{n}\right\|_{L_{n}^{2}}^{2}<\infty
$$

For $F \in \operatorname{Dom} D$, the Malliavin derivative is the stochastic process

$$
D F:[0, T] \times \mathbb{R} \times \Omega \longrightarrow \mathbb{R}
$$

defined by

$$
D_{z} F=\sum_{n=1}^{\infty} n I_{n-1}\left(f_{n}(z, \cdot)\right), \quad z \in[0, T] \times \mathbb{R},
$$

where the convergence of the series is in $L^{2}([0, T] \times \mathbb{R} \times \Omega, \mu \otimes \mathbb{P})$. The set Dom $D$ is a Hilbert space with the scalar product

$$
\langle F, G\rangle=\mathbb{E}[F G]+\mathbb{E}\left[\iint_{[0, T] \times \mathbb{R}} D_{z} F D_{z} G d \mu(z)\right]
$$

and $D$ is a closed operator from $\operatorname{Dom} D$ to $L^{2}([0, T] \times \mathbb{R} \times \Omega, \mu \otimes \mathbb{P})$.

Given the form of the measure $\mu$, for $f:([0, T] \times \mathbb{R})^{n} \rightarrow \mathbb{R}$ measurable, positive or $\mu^{\otimes n}$ integrable, we have 


$$
\begin{aligned}
& \int_{\left(\mathbb{R}_{+} \times \mathbb{R}\right)^{n}} f d \mu^{\otimes n} \\
& \left.=\int_{[0, T] \times([0, T] \times \mathbb{R})^{n-1}} f\left((t, 0), z_{1}, \ldots z_{n-1}\right)\right) d t d \mu^{\otimes(n-1)}\left(z_{1}, \ldots, z_{n-1}\right) \\
& \quad+\int_{[0, T] \times \mathbb{R}_{0} \times([0, T] \times \mathbb{R})^{n-1}} f\left(z_{1}, z_{2}, \ldots z_{n}\right) d \mu^{\otimes(n)}\left(z_{1}, z_{2}, \ldots, z_{n}\right),
\end{aligned}
$$

As a consequence, when $\sigma>0$ or $\nu \neq 0$, it is natural to consider two more spaces:

1. Let $\operatorname{Dom} D^{0}$ (if $\sigma>0$ ) be the set of $F \in L^{2}(\Omega)$ with decomposition $F=\sum_{n=0}^{\infty} I_{n}\left(f_{n}\right)$ such that

$$
\begin{aligned}
\sum_{n=1}^{\infty} n n ! \int_{[0, T] \times\left(R_{+} \times \mathbb{R}\right)^{n-1}} f^{2}\left((t, 0), z_{1}, \ldots, z_{n-1}\right) d t \\
\cdot d \mu^{\otimes(n-1)}\left(z_{1}, \ldots, z_{n-1}\right)<\infty,
\end{aligned}
$$

For such $F$ we can define the square integrable stochastic process

$$
D_{t, 0} F=\sum_{n=1}^{\infty} n I_{n-1}\left(f_{n}((t, 0), \cdot)\right)
$$

with convergence in $L^{2}([0, T] \times \Omega, d t \otimes \mathbb{P})$.

2. When $\nu \neq 0$, let Dom $D^{J}$ be the set of $F=\sum_{n=0}^{\infty} I_{n}\left(f_{n}\right)$ such that

$$
\sum_{n=1}^{\infty} n n ! \int_{R_{+} \times \mathbb{R}_{0} \times([0, T] \times \mathbb{R})^{n-1}} f_{n}^{2} d \mu^{\otimes n}<\infty
$$

and for $F$ satisfying this condition, define

$$
D_{z} F=\sum_{n=1}^{\infty} n I_{n-1}\left(f_{n}(z, \cdot)\right),
$$

convergence in $\left.L^{2}\left([0, T] \times \mathbb{R}_{0} \times \Omega, x^{2} d t d \nu(x) \otimes \mathbb{P}\right)\right)$.

When both $\sigma>0$ and $\nu \neq 0$, then $\operatorname{Dom} D=\operatorname{Dom} D^{0} \cap \operatorname{Dom} D^{J}$.

\subsection{Alternative definition of the derivative operator and practical rules}

For practical purposes, the above definitions are not very useful since, in general, the chaos expansion of a functional is not known, and therefore some rules to compute the derivative are needed. In the case of Brownian motion, through the definition of the derivative as a week derivative on the canonical space, a chain rule is proved (see, for example, Nualart [16]). For the case of 
the Poisson process, it is proved that the Malliavin derivative coincides with a difference operator on the canonical space (Nualart and Vives [18]). In our context we can proceed as following: Since the Brownian part and the jumps part of $X$ are independent, it can be constructed a canonical space of $X$ as a product space $\Omega_{W} \times \Omega_{J}, \mathcal{F}_{W} \otimes \mathcal{F}_{J}, \mathbb{P}_{W} \otimes \mathbb{P}_{J}$, where:

- $\left(\Omega_{W}, \mathcal{F}_{W}, \mathbb{P}_{W},\left\{W_{t}, t \in[0, T]\right\}\right)$ is the canonical Brownian process; that is $\Omega_{W}=\mathcal{C}([0, T])$ is the space of continuous functions on $[0, T]$, null at the origin, with the topology of the uniform convergence, $\mathcal{F}_{W}$ the Borel $\sigma$ algebra and $P_{W}$ the probability that makes the projections $W_{t}: \Omega_{W} \rightarrow \mathbb{R}$ a Brownian motion.

- $\left(\Omega_{J}, \mathcal{F}_{J}, \mathbb{P}_{J},\left\{J_{t}, t \in[0, T]\right\}\right)$ is the canonical pure jump Lévy process

$$
J_{t}=\iint_{(0, t] \times \mathbb{R}_{0}} x d \tilde{N}(t, x) .
$$

See [26] for a complete construction of this space, where we extend the ideas of Neveu [20] to a general Lévy process. Essentially, $\Omega_{J}$ is formed by infinite sequences $\omega=\left(\left(t_{1}, x_{1}\right),\left(t_{2}, x_{2}\right), \ldots\right) \in\left([0, T] \times \mathbb{R}_{0}\right)^{\mathbb{N}}$, such that for every $\varepsilon>0$, there is only a finite number of $\left(t_{i}, x_{i}\right)$ with $\left|x_{i}\right|>\varepsilon$, where the $t_{i}$ are the instants of jump and $x_{i}$ the size of the corresponding jump.

\section{Derivative $D_{t, 0}$}

In order to compute the derivative $D_{t, 0} F$ for $F \in L^{2}\left(\Omega_{W} \times \Omega_{J}\right)$ we can use the the isometry

$$
L^{2}\left(\Omega_{W} \times \Omega_{J}\right) \simeq L^{2}\left(\Omega_{W} ; L^{2}\left(\Omega_{J}\right)\right),
$$

and consider $F$ as element of $L^{2}\left(\Omega_{W} ; L^{2}\left(\Omega_{J}\right)\right)$ and apply the theory of Malliavin derivative of a Hilbert space valued random variable following Nualart [16, pag. 61]. Under some restrictions, it is proved (see Solé et al. [26]) that both derivatives coincide. This is proved in the following way: By definition, a $L^{2}\left(\Omega_{J}\right)$-valued smooth random variable has the form

$$
F=\sum_{i=1}^{n} G_{i} H_{i}
$$

where $G_{i}$ are ordinary Brownian smooth random variables and $H_{i} \in L^{2}\left(\Omega_{J}\right)$. Define the Malliavin derivative of $F$ as

$$
D_{t}^{W^{*}} F=\sum_{i=1}^{n} D_{t} G_{i} \otimes H_{i}
$$

where $D_{t}$ is the ordinary Malliavin derivative. This definition is extended to a subspace $\operatorname{Dom} D^{W^{*}}$. 
Proposition 1. Dom $D^{W^{*}} \subset \operatorname{Dom} D^{0}$, and for $F \in \operatorname{Dom} D^{W^{*}}$,

$$
D_{t}^{W^{*}} F=\sigma D_{t, 0} F \text {. }
$$

Idea of the proof.

First we consider the functionals of the form

$$
F=N\left(B_{1}\right) \cdots N\left(B_{m}\right) W\left(C_{1}\right) \cdots W\left(C_{k}\right)
$$

where $B_{1}, \ldots, B_{m} \in \mathcal{B}\left(\mathbb{R}_{+} \times \mathbb{R}_{0}\right)$ are pairwise disjoints, $0 \notin \bar{B}_{i}$, and $C_{1}, \ldots, C_{k}$ $\in \mathcal{B}\left(\mathbb{R}_{+}\right)$pairwise disjoints, with $\int_{C_{j}} d t<\infty$. Itô [9, Lemma 2], shows that such $F$ can be written as a sum of multiple integrals:

$$
F=I_{0}\left(f_{0}\right)+\cdots+I_{m+k}\left(f_{m+k}\right)
$$

and then the derivatives are easy to compute.

The final step is the extension of the the formula (6) to Dom $D^{W^{*}}$, that is done by a density argument.

Remark. Note that when there is no jump part, then $X_{t}=\sigma W_{t}$. Therefore, the derivative $D_{t, 0} F$ coincides with the classical Malliavin derivative, except in a factor $\sigma$, due to the fact that in $D_{t, x} F$ we are differentiating with respect to $\sigma W$.

From the above Proposition, we deduce the following rule of differentiation:

Proposition 2 (Chain rule). Let $F=f\left(Z, Z^{\prime}\right) \in L^{2}\left(\Omega_{W} \times \Omega_{J}\right)$ with $Z \in$ Dom $D^{W}$ and $Z^{\prime} \in L^{2}\left(\Omega_{J}\right)$, and $f(x, y)$ a continuously differentiable function with bounded partial derivatives in the variable $x$. Then $F \in \operatorname{Dom} D^{0}$ and

$$
D_{t, 0} F=\frac{1}{\sigma} \frac{\partial f}{\partial x}\left(Z, Z^{\prime}\right) D_{t}^{W} Z
$$

where $D^{W}$ is the Malliavin derivative in $\left(\Omega_{W}, \mathcal{F}_{W}, \mathbb{P}_{W}\right)$ and Dom $D^{W}$ its domain.

Derivative $D_{t, x}, x \neq 0$

Consider $\omega=\left(\omega^{W}, \omega^{J}\right) \in \Omega_{W} \times \Omega_{J}, \omega^{J}=\left(\left(t_{1}, x_{1}\right),\left(t_{2}, x_{2}\right), \ldots\right) \in([0, T] \times$ $\left.\mathbb{R}_{0}\right)^{\mathbb{N}}$, with the restrictions pointed out above.

Given $z=(t, x) \in[0, T] \times \mathbb{R}_{0}$, we introduce in $\omega^{J}$ a jump of size $x$ at instant $t$, and call the new element $\omega_{z}^{J}=\left(\left(t_{1}, x_{1}\right),\left(t_{2}, x_{2}\right), \ldots,(t, x), \ldots\right)$, and write $\omega_{z}=\left(\omega^{W}, \omega_{z}^{J}\right)$. For a random variable $F$, we define the translation operator

$$
\boldsymbol{\Psi}_{t, x} F=\frac{F\left(\omega_{t, x}\right)-F(\omega)}{x} .
$$

This operator has the following property ( Solé et al [26]) 
Proposition 3. Let $F \in L^{2}\left(\Omega_{W} \times \Omega_{J}\right)$ such that

$$
E\left[\iint_{[0, T] \times \mathbb{R}_{0}}\left(\boldsymbol{\Psi}_{z} F\right)^{2} \mu(d z)\right]<\infty .
$$

Then $F \in \operatorname{Dom} D^{J}$ and

$$
D_{z} F(\omega)=\boldsymbol{\Psi}_{z} F(\omega), \quad \mu \otimes \mathbb{P} \text { a.e. }(z, \omega) \in[0, T] \times \mathbb{R}_{0} \times \Omega .
$$

Idea of the proof.

We can restrict to a purely jump process. First, a compound Poisson process is considered; for this class of processes, as in the Poisson case, the multiple integral can be computed by each $\omega$. Also, for a functional $F$ with a finite chaos expansion, the translation operator can be computed explicitly. Then the equality of the derivative operator $D_{t, x}$ and the translation operator $\boldsymbol{\Psi}$ is checked on these functionals, and then extended to more general random variables. Finally, the result for a general purely jump Lévy process is obtained considering such process as limit a of compound processes. Of course, all the proof relies heavily on the structure of $\Omega_{J}$ that we have commented before.

As an example of the power of the chain rule and the increment quotient formula, in Sole et al. [27] there is the computation of the minimal quadratic hedging of an Asian option for a general jump-diffusion process, where the main difficulty is that the functional depends on the entire trajectory of the process, and not only on the final value as in the European case.

\subsection{The Skorohod integral}

Following the scheme of Nualart and Vives [18], we can define a creation operator (Skorohod integral) in the following way: Let $f \in L^{2}([0, T] \times \mathbb{R} \times$ $\left.\Omega, \mathcal{B}([0, T] \times \mathbb{R}) \otimes \mathcal{F}^{X}, \mu \otimes \mathbb{P}\right)$. There is a chaotic decomposition

$$
f(z)=\sum_{n=0}^{\infty} I_{n}\left(f_{n}(z, \cdot)\right)
$$

where $f \in L_{n+1}^{2}$ is symmetric in the $n$ last variables. Now, denote by $\widehat{f}_{n}$ the symmetrization in all $n+1$ variables. If

$$
\sum_{n=0}^{\infty}(n+1) !\left\|\widehat{f}_{n}\right\|_{L_{n+1}^{2}}^{2}<\infty
$$

define the Skorohod integral of $f$ by

$$
\delta(f)=\sum_{n=0}^{\infty} I_{n+1}\left(\widehat{f}_{n}\right)
$$

convergence in $L^{2}(\Omega)$. Denote by $\operatorname{Dom} \delta$ the set of $f$ that satisfy (8). The main properties of the operator $\delta$ are: 
1. Duality formula: A process $f \in L^{2}([0, T] \times \mathbb{R} \times \Omega, \mu \times \mathbb{P})$ belongs to $\operatorname{Dom} \delta$ if and only if there is a constant $C$ such that for all $F \in \operatorname{Dom} D$,

$$
\left|\mathbb{E} \iint_{[0, T] \times R} f(z) D_{z} F d \mu(z)\right| \leq C\left(\mathbb{E}\left[F^{2}\right]\right)^{1 / 2} .
$$

If $f \in \operatorname{Dom} \delta$, then $\delta(f)$ is the element of $L^{2}(\Omega)$ characterized by

$$
\mathbb{E}[\delta(f) F]=\mathbb{E} \iint_{[0, T] \times R} f(z) D_{z} F d \mu(z),
$$

for any $F \in \operatorname{Dom} D$.

2. Isometry: Denote by $\mathbb{L}^{1,2}$ the set of elements $f \in L^{2}([0, T] \times \mathbb{R} \times$ $\left.\Omega, \mathcal{B}([0, T] \times \mathbb{R}) \otimes \mathcal{F}^{X}, \mu \otimes \mathbb{P}\right)$ such that $f(z) \in \operatorname{Dom} D, \forall z \mu-$ a.e and that $D \cdot f(\cdot) \in L^{2}\left(([0, T] \times \mathbb{R})^{2} \times \Omega\right)$. In terms of the chaotic expression (7) of $f$, both conditions are equivalent to

$$
\sum_{n=1}^{\infty} n n !\left\|\widehat{f}_{n}\right\|_{L_{n+1}^{2}}^{2}<\infty
$$

and, in particular, this implies $\mathbb{L}^{1,2} \subset \operatorname{Dom} \delta$. For $f, g \in \mathbb{L}^{1,2}$,

$$
\begin{aligned}
E[\delta(f) \delta(g)]=\mathbb{E} \iint_{[0, T] \times \mathbb{R}} f(z) g(z) d \mu(z) & \\
& +\mathbb{E} \iint_{([0, T] \times R)^{2}} D_{z} f\left(z^{\prime}\right) D_{z} g\left(z^{\prime}\right) d \mu(z) d \mu\left(z^{\prime}\right) .
\end{aligned}
$$

3. Differentiability of $\delta$. Let $f \in \mathbb{L}^{1,2}$ such that $D_{z} f \in \operatorname{Dom} \delta, \forall z, \mu-$ a.e. Then $\delta(f) \in \operatorname{Dom} D$ and

$$
D_{z} \delta(f)=f(z)+\delta\left(D_{z} f\right), \forall z, \mu-\text { a.e. }
$$

\subsection{Integral respect to the random measure $M$ and Skorohod integral}

The random measure $M$, with the filtration $\left\{\mathcal{F}_{t}^{X}, t \in[0, T]\right\}$, induces a martingale-valued measure, and it can be defined a stochastic integral $\iint_{[0, T] \times \mathbb{R}} f(z) d M_{z}$, of a predictable process $f$ such that $E \iint_{[0, T] \times \mathbb{R}} f^{2}(z) \mu(d z)$ $<\infty$. (See Applebaum [1, Chapter 4]). For $f$ and $g \mu$-square integrable predictable processes

$$
\mathbb{E}\left[\iint_{[0, T] \times \mathbb{R}} f(z) d M_{z} \cdot \iint_{[0, T] \times \mathbb{R}} g(z) d M_{z}\right]=\mathbb{E}\left[\iint_{[0, T] \times \mathbb{R}} f(z) g(z) d \mu(z)\right] .
$$

An explicit expression for the integral $\iint_{[0, T] \times \mathbb{R}} f(z) d M_{z}$ is given in the following Proposition: 
Proposition 4. Let $f=\{f(z), z \in[0, T] \times \mathbb{R}\}$ be a predictable process such that $E \iint_{[0, T] \times \mathbb{R}} f^{2}(z) \mu(d z)<\infty$. Then

$$
\iint_{[0, T] \times \mathbb{R}} f(z) d M_{z}=\sigma \int_{0}^{T} f(t, 0) d W_{t}+\iint_{[0, T] \times \mathbb{R}_{0}} x f(t, x) d \tilde{N}(t, x) .
$$

As in the Brownian case, the Skorohod integral restricted to predictable processes coincides with the integral respect to the random measure $M$. The next theorem is a version of Privault [23, Proposition 11], Di Nunno et al. [6, Proposition 3.15], and Oksendal and Proske [21, Proposition 3.7]:

Theorem 5. Let $f \in L^{2}([0, T] \times \mathbb{R} \times \Omega)$ be a predictable process. Then $f \in$ Dom $\delta$ and

$$
\delta(f)=\iint_{[0, T] \times \mathbb{R}} f(z) d M_{z} .
$$

Sketch of the proof.

Consider first a simple predictable process $f$ of the form:

$$
f(z)=\beta \mathbf{1}_{(r, s] \times B}(z),
$$

where $0 \leq r<s \leq T, \beta$ is a bounded random variable $\mathcal{F}_{r}^{X}$ measurable, and $B \in \mathcal{B}(\mathbb{R})$. In order to prove that $f \in \operatorname{Dom}(\delta)$ and $\delta(f)=\iint_{[0, T] \times \mathbb{R}} f(z) d M_{z}$, by the duality relation (9) we need to check that for all $F \in \operatorname{Dom} D$, we have

$$
\mathbb{E}\left[\iint_{[0, T] \times R} f(z) D_{z} F d \mu(z)\right]=\mathbb{E}\left[F \iint_{[0, T] \times \mathbb{R}} f(z) d M_{z}\right] .
$$

By a density argument, it is enough to consider the case $F=I_{n}\left(\widetilde{f}_{n}\right)$, where $f_{n}=\mathbf{1}_{E_{1} \times \cdots \times E_{n}}$, with $E_{1} \ldots, E_{n} \in \mathcal{B}([0, T] \times \mathbb{R})$ pairwise disjoints with $\mu\left(E_{i}\right)<\infty$

\subsection{The Lévy process as a normal martingale: multiple integrals respect to $X$}

The Lévy process $X$ is a square integrable martingale with predictable quadratic variation $\langle X, X\rangle_{t}=c t$, where $c=\sigma^{2}+\int_{\mathbb{R}} x^{2} d \nu(x)$. Then, $X$ is a normal martingale in the sense of Dellacherie and Meyer [15, page 199]. If $g=\{g(t), t \in[0, T]\}$ is a (unidimensional) predictable process such that $E \int_{0}^{T} g(t)^{2} d t<\infty$, then $g$ will be integrable with respect to $X$. Moreover, $g(t) x$ is (two-parameter) predictable and it is also integrable with respect to $\widetilde{N}$, and

$$
\int_{0}^{T} g(t) d X_{t}=\sigma \int_{0}^{T} g(t) d W_{t}+\iint_{[0, T] \times \mathbb{R}_{0}} g(t) x d \widetilde{N}(t, x) .
$$


From Proposition 4 we deduce

$$
\int_{0}^{T} g(t) d X_{t}=\iint_{[0, T] \times \mathbb{R}} g(t) d M(t, x) .
$$

On the other hand, it can be defined a multiple integral respect to $X$ : see Meyer [15]. For $f \in L^{2}\left([0, T]^{n},(d t)^{n}\right)$, symmetric, we can consider $I_{n}^{X}(f)$, which coincides with $n$ ! times the iterated integral:

$$
I_{n}^{X}(f)=n ! \int_{0}^{T} \int_{0}^{t_{n}^{-}} \cdots \int_{0}^{t_{2}^{-}} f\left(t_{1}, \ldots, t_{n}\right) d X_{t_{n}} \cdots d X_{t_{2}} d X_{t_{1}} .
$$

The multiple integral with respect to $X, I_{n}^{X}$, coincides with the restriction of the multiple integral $I_{n}$ to $L^{2}\left([0, T]^{n}\right)$ : working first with elementary functions of the form $\mathbf{1}_{A_{1} \times \cdots \times A_{n}}, A_{1}, \ldots, A_{n} \in \mathcal{B}([0, T])$ pairwise disjoints, and by a density argument, it is proved that for $f \in L^{2}\left([0, T]^{n},(d t)^{n}\right)$, symmetric, we have $I_{n}^{X}(f)=I_{n}(f)$. This is a multivariate extension of (11).

\section{Malliavin calculus and Teugels martingales}

In the rest of the paper we will assume the condition of Nualart-Schoutens [17] (see also Schoutens [25]) about the exponential integrability of the Lévy measure, that is, there are $\varepsilon>0$ and $\lambda>0$ such that

$$
\int_{(-\varepsilon, \varepsilon)^{c}} \exp \{\lambda|x|\} \nu(d x)<\infty .
$$

This condition implies that the Lévy measure has moments of all order $\geq 2$, that $X_{t}$ has moments of all order, and that the polynomials are dense in $L^{2}\left(\mathbb{R}, P \circ X_{1}^{-1}\right)$.

\subsection{Teugels martingales}

Following Nualart and Schoutens [17] , consider the square integrable martingales, called Teugel martingales

$$
\begin{aligned}
Y_{t}^{(1)} & =X_{t}, \\
Y_{t}^{(n)} & =\sum_{0<s \leq t}\left(\Delta X_{s}\right)^{n}-m_{n} t, \quad n \geq 2,
\end{aligned}
$$

where $m_{n}=\int_{-\infty}^{\infty} x^{n} \nu(d x), n \geq 2$. Introduce also the martingales

$$
H_{t}^{(n)}=\sum_{j=1}^{n} a_{n j} Y_{t}^{(j)}, \quad n \geq 1,
$$


where the constants $a_{n j}$ are chosen in such a way that $a_{n n}=1$ and the martingales $H^{(n)}, n=1,2, \ldots$ are pairwise strongly orthogonal. Its predictable quadratic variation process is

$$
\left\langle H^{(n)}, H^{(m)}\right\rangle_{t}=\delta_{n m} q_{n} t,
$$

for some constants $q_{n}$; so they are normal martingales. The strong orthogonality of the martingales $H^{(n)}$ is equivalent to the existence of an orthogonal family of polynomials with respect to the measure

$$
d \eta(x)=\sigma^{2} d \delta_{0}(x)+x^{2} d \nu(x),
$$

Note that $d \mu(t, x)=d t d \eta(x)$. Specifically, the polynomials $p_{n}$ defined by

$$
p_{n}(x)=\sum_{j=1}^{n} a_{n j} x^{j-1}
$$

are orthogonal with respect to de measure $\eta$ :

$$
\int_{\mathbb{R}} p_{n}(x) p_{m}(x) d \eta(x)=0, \quad n \neq m .
$$

and the $q_{n}$ appearing in (13) is $q_{n}=\int_{\mathbb{R}} p_{n}^{2}(x) d \eta(x)$,

Proposition 6. Let $g=\{g(t), t \in[0, T]\}$ be a predictable process such that $\mathbb{E}\left[\int_{0}^{T} g^{2}(t) d t\right]<\infty$. Then the process $f(t, x)=g(t) p_{n}(x)$ is integrable with respect to $M$ and

$$
\int_{0}^{T} g(t) d H_{t}^{(n)}=\iint_{[0, T] \times \mathbb{R}} g(t) p_{n}(x) d M(t, x) .
$$

Proof.

For $n=1$, we have $p_{1}(x)=1, H^{(1)}=Y^{(1)}=X$, and then (14) is the formula (11).

For $n \geq 2$, the process $g(t) x^{n}$ is predictable and

$$
\mathbb{E}\left[\iint_{[0, T] \times \mathbb{R}} g^{2}(t) x^{2 n} d t d \nu(x)\right]<\infty .
$$

Therefore it is integrable with respect to the random measure $\widetilde{N}(t, x)$ and

$$
\int_{0}^{T} g(t) d Y_{t}^{(n)}=\iint_{[0, T] \times \mathbb{R}_{0}} g(t) x^{n} d \widetilde{N}(t, x)=\iint_{[0, T] \times \mathbb{R}} g(t) x^{n-1} d M(t, x)
$$

It follows that

$$
\begin{aligned}
\int_{0}^{T} g(t) d H_{t}^{(n)} & =\sum_{j=1}^{n} a_{n, j} \int_{0}^{T} g(t) d Y_{t}^{(j)}=\sum_{j=1}^{n} a_{n, j} \iint_{[0, T] \times \mathbb{R}} g(t) x^{j-1} d M(t, x) \\
& =\iint_{[0, T] \times \mathbb{R}} g(t) p_{n}(x) d M(t, x) .
\end{aligned}
$$


Note that in particular,

$$
H_{t}^{(n)}=\iint_{[0, t] \times \mathbb{R}} p_{n}(x) d M(s, x) .
$$

\subsection{The chaotic representation of Nualart and Schoutens}

The chaotic representation of Nualart and Schoutens uses iterated integrals of the form

$$
\begin{aligned}
J_{n}^{\left(i_{1}, \ldots, i_{n}\right)}(g) & =\int_{0}^{T}\left(\int_{0}^{t_{n}-} \ldots\right. \\
& \left.\left(\int_{0}^{t_{2}-} g\left(t_{1}, \ldots, t_{n}\right) d H^{\left(i_{1}\right)}\left(t_{1}\right)\right) \cdots d H^{\left(i_{n-1}\right)}\left(t_{n-1}\right)\right) d H^{\left(i_{n}\right)}\left(t_{n}\right),
\end{aligned}
$$

for $g \in L^{2}\left([0, T]^{n}\right)$. For different indices, these integrals are orthogonal (see Leon et al [12]): Given $f \in L^{2}\left([0, T]^{n}\right)$ and $g \in L^{2}\left([0, T]^{m}\right)$,

$$
\begin{aligned}
& E\left[J_{n}^{\left(i_{1}, \ldots, i_{n}\right)}(f) J_{m}^{\left(j_{1}, \ldots, j_{m}\right)}(g)\right] \\
& \quad=\left\{\begin{array}{cc}
q_{i_{1}} \cdots q_{i_{n}} \int_{\Sigma_{n}} f\left(t_{1}, \ldots, t_{n}\right) g\left(t_{1}, \ldots, t_{n}\right) d t_{1} \cdots d t_{n}, \\
\text { if } n=m \text { and }\left(i_{1}, \ldots, i_{n}\right)=\left(j_{1}, \ldots, j_{n}\right), \\
0, \quad \text { otherwise },
\end{array}\right.
\end{aligned}
$$

where $\Sigma_{n}=\left\{\left(t_{1}, \ldots, t_{n}\right) \in \mathbb{R}_{+}^{n}: 0<t_{1}<t_{2}<\cdots<t_{n} \leq T\right\}$ is the positive simplex of $[0, T]^{n}$.

Nualart and Schoutens [17, Theorem 5] prove that every square integrable random variable $F$ has a representation of the form

$$
F=E F+\sum_{n=1}^{\infty} \sum_{i_{1}, \ldots, i_{n} \geq 1} J_{n}^{\left(i_{1}, \ldots, i_{n}\right)}\left(g_{i_{1}, \ldots, i_{n}}\right),
$$

where $g_{i_{1}, \ldots, i_{n}} \in L^{2}\left([0, T]^{n}\right)$. We will assume that the functions $g_{i_{1}, \ldots, i_{n}}$ in that representation are symmetric.

The next proposition gives the relationship between the iterated integral and the multiple integral and it is an extension of formulas (12) and (14):

Proposition 7. Let $g \in L^{2}\left([0, T]^{n}\right)$. Then

$$
J_{n}^{\left(i_{1}, \ldots, i_{n}\right)}(g)=I_{n}\left(g\left(t_{1}, \ldots, t_{n}\right) \mathbf{1}_{\Sigma_{n}}\left(t_{1}, \ldots, t_{n}\right) p_{i_{1}}\left(x_{1}\right) \cdots p_{i_{n}}\left(x_{n}\right)\right) .
$$




\subsection{Malliavin derivative in the $k$-th direction}

Let $g \in L^{2}\left([0, T]^{n}\right)$ and $k \geq 1$. Leon et al. [12] define the derivative of $J_{n}^{\left(i_{1}, \ldots, i_{n}\right)}(g)$ in the $k$-th direction as the process

$$
D_{t}^{(k)} J_{n}^{\left(i_{1}, \ldots, i_{n}\right)}(g)=\sum_{\ell=1}^{n} \mathbf{1}_{\left\{i_{\ell}=k\right\}} J_{n-1}^{\left(i_{1}, \ldots, \hat{i}_{\ell}, \ldots, i_{n}\right)}\left(g(\underbrace{\ldots}_{\ell-1}, t, \cdots) \mathbf{1}_{\Sigma_{n}^{(\ell)}(t)}(\cdot)\right),
$$

where

$$
\begin{aligned}
& \Sigma_{n}^{(\ell)}(t)= \\
& \quad\left\{\left(t_{1}, \ldots, \widehat{t}_{\ell}, \ldots, t_{n}\right) \in \Sigma_{n-1}: 0<t_{1}<\cdots<t_{\ell-1}<t \leq t_{\ell+1}<\cdots<t_{n}\right\}
\end{aligned}
$$

and $\hat{i}$ means that the $i$-th index is omitted. This definition is extended in a natural way to the subspace of $L^{2}(\Omega)$ :

$$
\begin{aligned}
& \operatorname{Dom} D^{(k)}=\left\{F \in L^{2}(\Omega), F=E F+\sum_{n=1}^{\infty} \sum_{i_{1}, \ldots, i_{n} \geq 1} J_{n}^{\left(i_{1}, \ldots, i_{n}\right)}\left(g_{i_{1}, \ldots, i_{n}}\right):\right. \\
& \left.\sum_{n=1}^{\infty} \frac{1}{n !} \sum_{i_{1}, \ldots, i_{n} \geq 1} \sum_{\ell=1}^{n} \mathbf{1}_{\left\{i_{\ell}=k\right\}} q_{i_{1}} \cdots \widehat{q_{i_{\ell}}} \cdots q_{i_{n}}\left\|g_{i_{1}, \ldots, i_{n}}\right\|_{L^{2}\left([0, T]^{n}\right)}^{2}<\infty\right\} .
\end{aligned}
$$

From the relationship between the kernels of the representations (3) and (16) (see Benth et al. [2, Formula 3.22]), we obtain that $F$ with the representation

$$
F=E F+\sum_{n=1}^{\infty} \sum_{i_{1}, \ldots, i_{n} \geq 1} J_{n}^{\left(i_{1}, \ldots, i_{n}\right)}\left(g_{i_{1}, \ldots, i_{n}}\right)
$$

belongs to $\operatorname{Dom} D$ if and only if

$$
\sum_{n=1}^{\infty} \frac{1}{(n-1) !} \sum_{i_{1}, \ldots, i_{n} \geq 1} q_{i_{1}} \cdots q_{i_{n}}\left\|g_{i_{1}, \ldots, i_{n}}\right\|_{L^{2}\left([0, T]^{n}\right)}^{2}<\infty .
$$

The following Proposition is based on Benth et al. [2, Proposition 3.8] and Di Nunno et al. [6, Remark 4.3]:

\section{Proposition 8.}

$$
\begin{aligned}
& F \in \operatorname{Dom} D \text { if and only if } F \in \operatorname{Dom} D^{(k)}, \\
& \qquad \text { for every } k \geq 1 \text {, and } \sum_{n=1}^{\infty} q_{n}\left\|D^{(n)} F\right\|^{2}<\infty .
\end{aligned}
$$

In that case, 


$$
D_{t, x} F=\sum_{n=1}^{\infty} p_{n}(x) D_{t}^{(n)} F
$$

and

$$
\begin{aligned}
D_{t}^{(n)} F= & \frac{1}{\int_{\mathbb{R}} p_{n}^{2}(x) d \eta(x)} \int_{\mathbb{R}} p_{n}(x) D_{t, x} F d \eta(x) \\
= & \frac{1}{p_{n}^{2}(0) \sigma^{2}+\int_{\mathbb{R}} x^{2} p_{n}^{2}(x) d \nu(x)} \\
& \left(p_{n}(0) D_{t, 0} F+\int_{\mathbb{R}} p_{n}(x) D_{t, x} F x^{2} d \nu(x)\right) .
\end{aligned}
$$

Proof. The equivalence between the domains of $D$ and $D^{(k)}$ is a consequence of (19) and (20).

The relationship (21), is proved by checking the formula for an iterated integral $J_{n}^{\left(i_{1}, \ldots, i_{n}\right)}(g)$, where $g\left(t_{1}, \ldots, t_{n}\right)=g_{1}\left(t_{1}\right) \cdots g_{n}\left(t_{n}\right)$, with $\int_{0}^{T} g_{i}^{2}(t) d t<$ $\infty$, and extending the equality by linearity and continuity to an arbitrary $F \in \operatorname{Dom} D$.

The second formula is deduced from the orthogonality of the polynomials $p_{n}(x)$.

\subsection{Skorohod integrals}

Following Davis and Johanson [3] we define the Skorohod integral in the direction $k$ as the adjoint of the densely defined operator $D^{(k)}$ : Let $f=\{f(t), t \in$ $[0, T]\} \in L^{2}([0, T] \times \Omega, d t \otimes \mathbb{P})$ be a stochastic process such that there is a constant $C$ such that for every $F \in \operatorname{Dom} D^{(k)}$,

$$
\left|\mathbb{E}\left[\int_{0}^{T}\left(f(t) D_{t}^{(k)} F\right)\right]\right| \leq C\left(\mathbb{E}\left[F^{2}\right]\right)^{1 / 2} .
$$

Then, we say that $f$ is Skorohod integrable in the $k$ direction, and define $\delta^{(k)}$ as the element of $L^{2}(\Omega)$ such that

$$
\mathbb{E}\left[\int_{0}^{T}\left(f(t) D_{t}^{(k)} F\right)\right]=\mathbb{E}\left[\delta^{(k)}(f) F\right], \text { for any } F \in \operatorname{Dom} D^{(k)} .
$$

Denote by $\operatorname{Dom} \delta^{(k)}$ the set of processes that satisfy (22).

The following Proposition is a consequence of Proposition 8 and the characterization (9) of Dom $\delta$.

Proposition 9. Let $f \in \operatorname{Dom} \delta^{(\mathrm{k})}$. Then $f(t) p_{k}(x) \in \operatorname{Dom} \delta$ and

$$
\delta^{(k)}(f(t))=\delta\left(f(t) p_{k}(x)\right) .
$$




\subsection{Clark-Ocone-Haussmann formula}

There are two Clark-Ocone-Haussmann formulas, one with the derivative $D_{t, x} F$ and the other one with $D_{t}^{(n)} F$. Thanks to Proposition 8 it is easy to go from one to the other. The next Theorem appears in Benth et al. [2], Di Nunno et al. [6], [7] and Oksendal and Proske [21]. We stated it in our context:

Theorem 10 (Clark-Ocone-Haussmann formula, twoparameter derivative.). Let $F \in L^{2}(\Omega)$ such that $F \in \operatorname{Dom} D$ and $\mathbb{E} \iint_{[0, T] \times \mathbb{R}}\left(D_{z} F\right)^{2} d \mu(z)<\infty$. Then

$$
F=\mathbb{E}[F]+\sigma \int_{0}^{T}{ }^{\mathrm{p}}\left(D_{t, 0} F\right) d W_{t}+\iint_{[0, T] \times \mathbb{R}_{0}}{ }^{\mathrm{p}}\left(D F_{t, x}\right) x d \widetilde{N}(t, x),
$$

where ${ }^{\mathrm{p}} G$ means the predictable projection of a stochastic process $G$.

Idea of the proof.

The proof is divided in two steps: First, we look for a process $h(x, t)$ such that

$$
F=\mathbb{E}[F]+\delta(h) .
$$

From the experience with the Brownian motion (Nualart [16]) and with normal martingales (Ma, Protter and Sanmartin [14]), we check that $h(t, x)=$ $\mathbb{E}\left[D_{t, x} F / \mathcal{F}_{t}\right]$ satisfies this condition.

In the second step, we look for a predictable process $h^{\prime}(t, x)$ such that $\delta(h)=\delta\left(h^{\prime}\right)$. Also, by previous experience, we take $h^{\prime}(t, x)={ }^{\mathrm{p}} D_{t, x} F$ and then we apply Theorem 5 and Proposition 4.

\section{Remarks on the predictable projection.}

1. The notion of Predictable Projection of a stochastic process indexed by $t \geq 0$ can be extended easily to our context for a two-parameter process. Let $Y=\left\{Y_{t, x},(t, x) \in[0, T] \times \mathbb{R}\right\}$ be a positive or bounded measurable process. There exists a predictable process $Z=\left\{Z_{t, x},(t, x) \in[0, T] \times \mathbb{R}\right\}$ such that for every predictable stopping time $\tau$

$$
Z_{\tau, x}=\mathbb{E}\left[Y_{\tau, x} / \mathcal{F}_{\tau-}\right] \text { on }\{\tau<\infty\}
$$

This result is based in the same ideas as in the ordinary case (see, for example, Dellacherie et Meyer [4], pp. 114-115).

2. To compute the projective projection of a process is, in general, not easy. However, one can skip this task if one can find a predictable process $h$ such that

$$
h(t, x)=\mathbb{E}\left[D_{t, x} F / \mathcal{F}_{t}^{X}\right], \quad \forall(t, x, \omega)-\mu \otimes \mathbb{P} \text { a.e. }
$$

Fortunately, this happens in many applications (see Solé el al. [27]). 
From Proposition 8 and Theorem 10 we can get a new proof of the ClarkOcone-Haussman formula using the derivatives in the direction $k$ (Leon et al. $[12$, Theorem 1.8]):

Theorem 11 (Clark-Ocone-Haussmann formula, derivatives in the $k$-th direction.). Let $F \in L^{2}(\Omega)$ such that $F \in \operatorname{Dom} D$ and $\mathbb{E} \iint_{[0, T] \times \mathbb{R}}\left(D_{z} F\right)^{2} d \mu(z)$ $<\infty$. Then

$$
F=\mathbb{E}[F]+\sum_{k=1}^{\infty} \int_{0}^{T}{ }^{\mathrm{p}}\left(D_{t}^{(k)} F\right) d H_{t}^{(k)} .
$$

\section{References}

1. Applebaum, D., Lévy Processes and Stochastic Calculus. Cambridge University Press, Cambridge (2004).

2. Benth, F. E., Di Nunno, G., Løkka, A., Øksendal, B. and Proske, F., Explicit representations of the minimal variance protfolio in markets driven by Lévy processes. Mathematical Finanace 13 (2003) 55-72.

3. Davis, M. H. A. And Johanson, M. P., Malliavin Monte Carlo Greeks for jump diffusions. Stoch. Proc. and their Appl. 116 (2006) 101-129.

4. Dellacherie, C. et Meyer, P. A., Probabilités et Potentiel. Theorie des Martingales. Hermann, Paris (1982).

5. Dellacherie, C. et Meyer, P. A., Probabilités et Potentiel. Processus de Markov (fin). Compléments de calcul stochastique. Hermann, Paris (1992).

6. Di Nunno, G., Øksendal, B. And Proske, F., White Noise Analysis for Lévy processes. Journal of Functional Analysis 206 (2004) 109-148.

7. Di Nunno, G., Meyer-Brandis, Th., Øksendal, B. and Proske, F., Malliavin calculus and anticipative Itô formulae for Lévy processes. Preprint series in Pure Mathematics, University of Oslo (2004) 16.

8. Iто̂, K., Multiple Wiener integral. J. Math. Soc. Japan 3 (1951) 157-169.

9. Iтô, K., Spectral type of the shift transformation of differential processes with stationary increments. Trans. Am. Math. Soc. 81 (1956) 252-263

10. LeE, Y.-J. AND Shin, H.-H., The product formula of multiple Lévy-Itô integrals. Bull. Inst. Math. Acad. Sinica 32 (2004) 71-95.

11. LeE, Y.-J. AND Shin, H.-H., Analysis of generalized Lévy white noise functionals. Journal of Functional Analysis 211 (2004) 1-70.

12. Leon, J., Solé, J.L, Utzet, F. And Vives, J., On Lévy processes, malliavin calculus and market models with jumps. Finance and Stochastics 6 (2002) $197-225$

13. LøKKA, A., Martingale representations and functionals of Lévy processes. Preprint series in Pure Mathematics, University of Oslo (2001) 21.

14. Ma, J, Protter, P. And San Martin, J., Anticipating integrals for a class of martingales. Bernoulli 4 (1998) 81-114

15. Meyer, P. A., Un cours sur les integrales stochastiques, In: SÚminaire de ProbailitÚs X, Lecture Notes in Mathematics, 511, 245-400, Springer, New York, 1976.

16. Nualart, D., The Malliavin Calculus and Related topics . Springer, Berlin (1995). 
17. Nualart, D. And Schoutens, W., Chaotic and predictable representation for Lévy processes. Stochastic Processes and their Applications 90 (2000) 109-122.

18. Nualart, D. And Vives, J., Anticipative calculus for the Poisson Process based on the Fock space. In: Séminaire de Probabilités XXIV (Lecture Notes in Mathematics, 1426), Springer, Berlin Heildelberg New York: Springer 154165( 1990).

19. Nualart, D. And Vives, J., A duality formula on the Poisson space and some applications. In: Proceedings of the Acona Conference on Stochastic Analysis. (Progress in Probability). Birkhauser (1995).

20. Neveu, J., Processus Pontuels. In: École d'Eté de Probabilités de Saint Flour, VI, (Lecture Notes in Mathematics, 598), Springer, Berlin (1977).

21. Øksendal, B. and Proske, F., White Noise of Poisson random measure. Potential Analysis 21 (2004) 375-403.

22. Picard, J., , On the existence of smooth densities for jump process, Probab. Theory Relat. Fields 105 (1996) 481-511.

23. Privault, N., An extension of stochastic calculus to certain non-Markovian processes Preprint 49, Universite d'Evry, (1997) http://www . maths. unive-evry.fr/prepubli/49.ps.

24. Sato, K., Lévy Processes and Infinitely Divisible Distributions. Cambridge University Press, Cambridge (1999).

25. Schoutens, W., Stochastic Processes and Orthogonal Polynomial, Lecture Notes in Statistics, 146, Springer, New York, 2000.

26. Solé, J., Utzet, F. And Vives, J., Canonic Lévy process and Malliavin Calculus. Stoch. Proc. and their Appl., to appear (2006).

27. Solé, J., Utzet, F. And Vives, J., Quadratic hedging of Asian options in jump diffusion models via Malliavin Calculus. Preprint (2006).

28. YABlonski, A., The calculus of variations for processes with independent increments, Rocky Mountain Journal of Mathematics, to appear (2006). 